\title{
The E3 ubiquitin ligases $\beta$-TrCP and FBXW7 cooperatively mediates GSK3-dependent Mcl-1 degradation induced by the Akt inhibitor API-1, resulting in apoptosis
}

Hui Ren ${ }^{1,2+}$, Junghui $\mathrm{Koo}^{2 \dagger}$, Baoxiang Guan ${ }^{2 \dagger}$, Ping Yue ${ }^{2}$, Xingming Deng ${ }^{3}$, Mingwei Chen ${ }^{{ }^{*}}$, Fadlo R Khuri ${ }^{2}$ and Shi-Yong Sun ${ }^{2^{*}}$

\begin{abstract}
Background: The novel Akt inhibitor, API-1, induces apoptosis through undefined mechanisms. The current study focuses on revealing the mechanisms by which API-1 induces apoptosis.

Results: API-1 rapidly and potently reduced the levels of Mcl-1 primarily in API-1-senstive lung cancer cell lines. Ectopic expression of Mcl-1 protected cells from induction of apoptosis by API-1. API-1 treatment decreased the half-life of Mcl-1, whereas inhibition of the proteasome with MG132 rescued Mcl-1 reduction induced by API-1. API-1 decreased Mcl-1 levels accompanied with a rapid increase in Mcl-1 phosphorylation (S159/T163). Moreover, inhibition of GSK3 inhibited Mcl-1 phosphorylation and reduction induced by API-1 and antagonized the effect of API-1 on induction of apoptosis. Knockdown of either FBXW7 or $\beta$-TrCP alone, both of which are E3 ubiquitin ligases involved in Mcl-1 degradation, only partially rescued Mcl-1 reduction induced by API-1. However, double knockdown of both E3 ubiquitin ligases enhanced the rescue of API-1-induced Mcl-1 reduction.
\end{abstract}

Conclusions: API-1 induces GSK3-dependent, $\beta$-TrCP- and FBXW7-mediated MCl-1 degradation, resulting in induction of apoptosis.

Keywords: API-1, GSK3, Mcl-1, E3 ubiquitin ligase, Apoptosis, Lung cancer

\section{Background}

API-1 (4-amino-5,8-dihydro-5-oxo-8- $\beta$-D-ribofuranosylpyrido[2,3- $d$ ] pyrimidine-6-carboxamide) is a recently identified novel Akt inhibitor. It inhibits Akt activity through binding to the pleckstrin homology domain of Akt and blocking its membrane translocation [1]. API-1 possesses promising anticancer activity, evidenced by its ability to suppress cell growth, induce apoptosis and inhibit the growth of cancer xenografts, particularly those with activated Akt, in nude mice [1]. We have recently shown that API-1 facilitates c-FLIP degradation, induces apoptosis

\footnotetext{
* Correspondence: chenmingwei@mail.xjtu.edu.cn; ssun@emory.edu

${ }^{\dagger}$ Equal contributors

'Department of Respiration, First Affiliated Hospital of Medical College of

Xi'an Jiaotong University, Xi'an, Shaanxi, P R China

${ }^{2}$ Department of Hematology and Medical Oncology, Emory University School

of Medicine and Winship Cancer Institute, Atlanta, Georgia, USA

Full list of author information is available at the end of the article
}

and enhances tumor necrosis factor-related apoptosisinducing ligand (TRAIL)-induced apoptosis in human nonsmall cell lung cancer (NSCLC) cells [2]. c-FLIP degradation clearly contributes to the enhancement of TRAIL-induced apoptosis by API-1 [2]. However, the mechanisms by which API-1 induces apoptosis in cancer cells and the additional mechanisms accounting for API-1-mediated augmentation of TRAIL-induced apoptosis are largely unknown.

In addition to the extrinsic death receptor-mediated apoptotic pathway, which is characterized by the oligomerization of cell surface death receptors and activation of caspase-8, the intrinsic apoptotic pathway that involves the disruption of mitochondrial membranes, release of cytochrome $\mathrm{c}$ and activation of caspase- 9 is another critical apoptotic mechanism [3]. It is known that the intrinsic apoptotic pathway is negatively regulated by anti-apoptotic Bcl-2 family members (e.g., $\mathrm{Mcl}-1, \mathrm{Bcl}-2$ and $\mathrm{Bcl}-\mathrm{X}_{\mathrm{L}}$ ) and inhibitor of 
apoptosis proteins (IAPs; e.g., survivin). In general, downregulation of these anti-apoptotic proteins can trigger apoptosis or augment TRAIL-induced apoptosis [4-6].

Among the anti-apoptotic Bcl-2 family members, Mcl-1 is known to be a short-lived protein that undergoes ubiquitination/proteasome-mediated degradation [7]. One degradation mechanism involves glycogen synthase kinase 3 (GSK3), which phosphorylates Mcl-1 at S159, triggering Mcl-1 degradation [8,9]. It has been suggested that Mcl-1 phosphorylation at S159 facilitates the association of Mcl-1 with the E3 ligase $\beta$-transducin repeats-containing protein $(\beta-\operatorname{TrCP})$, resulting in $\beta$-TrCP-mediated ubiquitination and degradation of Mcl-1. Recently two studies have suggested that phosphorylation at S159 enhances the association of Mcl-1 with the E3 ligase F-box/WD repeatcontaining protein 7 (FBXW7), resulting in FBXW7-mediated ubiquitination and degradation of Mcl-1 $[10,11]$.

In this study, we focused on revealing mechanisms by which API-1 induces apoptosis of cancer cells and uncovered GSK3-dependent Mcl-1 degradation as a critical mechanism accounting for induction of apoptosis by API-1. This mechanism also contributes to augmentation of TRAIL-induced apoptosis by API-1.

\section{Methods \\ Reagents}

API-1 (NSC177233) was obtained from the National Cancer Institute (Bethesda, MD). MK2206 was purchased from Active Biochem (Maplewood, NJ). They were dissolved in DMSO and stored at $-80^{\circ} \mathrm{C}$. Soluble recombinant human TRAIL was purchased from PeproTech, Inc. (Rocky Hill, NJ). The proteasome inhibitor MG132, the protein synthesis inhibitor cycloheximide (CHX) and the GSK3 inhibitor SB216763 were purchased from Sigma Chemical Co. (St. Louis, MO). The neddylation inhibitor MLN4924 was provided by Millennium Pharmaceuticals, Inc (Cambridge, MA). Expression plasmids in $\mathrm{pCI}$ vector carrying wild-type and mutant (S159A) human Mcl-1 were provided by Dr. X. Deng (Emory University, Atlanta, GA). Mouse monoclonal survivin and caspase- 8 antibodies and rabbit polyclonal Bim, caspase- 9 and PARP antibodies were purchased from Cell Signaling Technology (Danvers, MA). Mouse monoclonal caspase-3 antibody was purchased from Imgenex (San Diego, CA). Rabbit polyclonal Mcl-1, Bad, Bcl- $\mathrm{X}_{\mathrm{L}}$ and SKP1 and mouse monoclonal Bcl-2, Cul-1 and $\alpha$-tubulin antibodies were purchased from Santa Cruz Biotechnology, Inc. (Santa Cruz, CA). GSK3 $\alpha / \beta$ antibody was purchased from Upstate/Millipore (Billerica, MA). Mouse monoclonal Bax and rabbit polyclonal glyceraldehyde 3phosphate dehydrogenase (GAPDH) antibodies were purchased from Trevigen Inc. (Gaithersburg, MD). Both polyclonal and monoclonal actin antibodies were purchased from Sigma Chemical Co.

\section{Cell lines and cell culture}

The human NSCLC cell lines used in this study including those stably expressing ectopic Mcl-1 or survivin were described previously [12-14]. A549 cells were recently authenticated by Genetica DNA Laboratories, Inc. (Cincinnati, $\mathrm{OH}$ ) through analyzing short tandem repeat DNA profile; other cell lines have not been authenticated. HCT116/wild type (WT) and HCT116/FBXW7$\mathrm{KO}$ cell lines were kindly provided by Dr. B. Vogelstein (Johns Hopkins University School of Medicine, Baltimore, MA). These cell lines were grown in monolayer culture in RPMI 1640 medium or McCoy's medium supplemented with glutamine and $5 \%$ fetal bovine serum at $37^{\circ} \mathrm{C}$ in a humidified atmosphere consisting of $5 \% \mathrm{CO}_{2}$ and $95 \%$ air.

\section{Cell survival and apoptotic assays}

Cells were seeded in 96-well cell culture plates and treated the next day with the given agents. Viable cell numbers were determined using sulforhodamine $B$ (SRB) assay as described previously [15]. Combination index (CI) for drug interaction (e.g., synergy) was calculated using the CompuSyn software (ComboSyn, Inc.; Paramus, NJ). Apoptosis was evaluated with an annexin V-PE apoptosis detection kit (BD Biosciences; San Jose, $\mathrm{CA}$ ) according to the manufacturer's instructions. We also detected caspases and PARP cleavage by Western blot analysis as described below as additional indicators of apoptosis.

\section{Western blot analysis}

Preparation of whole-cell protein lysates and Western blot analysis were described previously $[16,17]$.

\section{Small interfering RNA (siRNA) and transfection}

GSK3 $\alpha / \beta$ siRNA (\#6301) was purchased from Cell Signaling Technology. FBXW7 siRNA that targets the sequence of 5'-AACACAAAGCTGGTGTGTGCA-3' [18] was synthesized from Qiagen (Valencia, CA) and used in our previous study [13]. Cullin 1 (Cul1; sc-35126), SKP1 (sc-29482) and $\beta$-TrCP (sc-37178) siRNAs were purchased from Santa Cruz Biotechnology, Inc. siRNA transfection was performed with HiPerFect transfection reagent (Qiagen) or Lipofectamine 2000 (Invitrogen) following the manufacturer's instructions.

\section{Reverse transcription-PCR (RT-PCR)}

To confirm knockdown efficiencies of $\beta$-TrCP and FBXW7 siRNA, Control, $\beta$-TrCP, FBXW7 and $\beta$-TrCP plus FBXW7 siRNAs were transfected into H1299 cells with Lipofectamine 2000. After $48 \mathrm{~h}$, total RNA was then prepared from the cells by Trizol (Sigma). Reverse transcription was performed with iScript select cDNA synthesis kit (Bio-Rad; Hercules, CA), followed with PCR using primers as follows: $\beta$-TrCP, $5^{\prime}$-CCCCAACTGACAT 
TACCC-3' (forward) and 5'-TCGAATACAACGCACC AA-3' (reverse) [19]; FBXW7, 5'-AAAGAGTTGTTAG CGGTTCTCG-3' (forward) and 5'-CCACATGGATAC CATC AAACTG-3' (reverse) [20]; GAPDH, 5'-TGAT GACATCAAGAAGGTGGTGAAG-3' (forward) and 5' TCCTTGGAGGCCATGTGGGCCAT-3' (reverse) [21]. Using the same assay, Mcl-l mRNA expression in cells exposed to API-1 was detected with the following primers: 5'-TAAGGACAAAACGGGACTGG-3' (forward) and 5'ACCAGCTCCTACTCCAGCAA-3' (reverse) [22].

\section{Results}

API-1 induces Mcl-1 reduction in API-1 sensitive NSCLC cell lines

Human NSCLC cell lines exhibited varied sensitivities to API-1 as evaluated with the SRB assay after a 3-day incubation (Figure 1A). Among them, H1299, H522 and A549 were sensitive to API-1, whereas H226 and H1792 were quite resistant to API-1. Since our previous work investigated the effects of API-1 on the expression of several proteins (e.g., c-FLIP, DR4 and DR5) involved in the extrinsic apoptotic pathway [2], we focused our current study on determining the effects of API-1 on modulation of the levels of several proteins (e.g., Mcl-1 and survivin) involved in regulation of the intrinsic apoptotic pathway. In H1299 cells, API-1 decreased the levels of Mcl-1 and survivin at even $2.5 \mu \mathrm{M}$ and the levels of Bcl-2 and Bcl- $\mathrm{X}_{\mathrm{L}}$ at $10 \mu \mathrm{M}$ with no apparent increase in the levels of the pro-apoptotic proteins, Bax, Bad and Bim (Figure 1B). Moreover, we found that API1 decreased Mcl-1 levels at $4 \mathrm{~h}$, survivin levels at $8 \mathrm{~h}$ and Bcl-2 levels at $12 \mathrm{~h}$ post treatment (Figure $1 \mathrm{C}$ ), indicating that Mcl-1 reduction is a rapid event ahead of survivin and Bcl-2 decrease in the process of API-1induced apoptosis. By further comparing effects of API1 on reducing Mcl-1 and survivin in 4 more NSCLC cell lines with different sensitivities to API-1, We found that API-1 reduced Mcl-1 levels effectively in $\mathrm{H} 522$ and A549 cells, which are sensitive to API-1, but only minimally in H226 and H1792 cells, which are insensitive to API-1. We detected survivin reduction in all four cell lines post API-1 treatment regardless of their sensitivities to

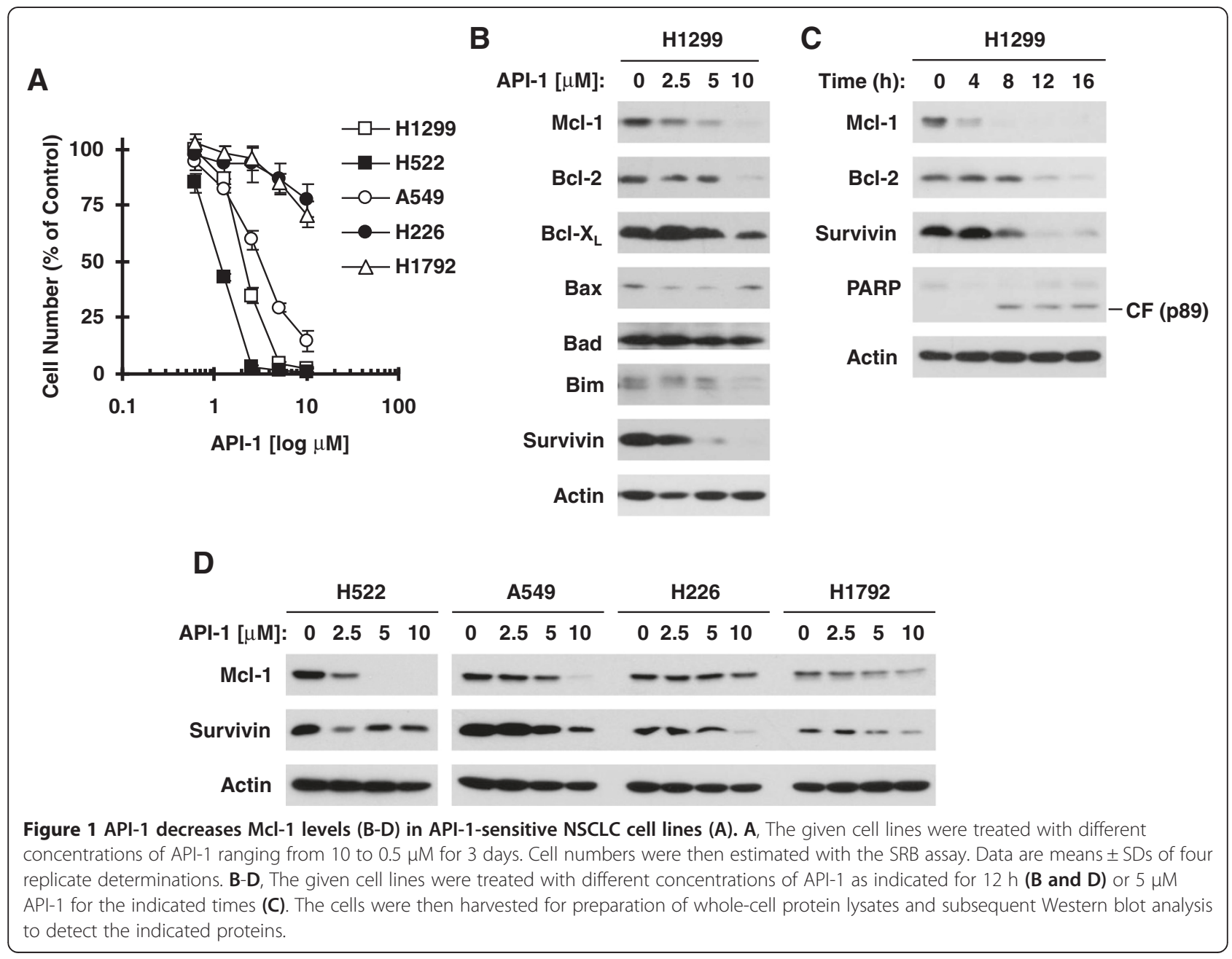


API-1 (Figure 1D). These data emphasize the relevance and importance of $\mathrm{Mcl}-1$ reduction in cell response to API-1.

Enforced expression of ectopic Mcl-1 protects NSCLC cells from induction of apoptosis by API-1 or API-1 combined with TRAIL

We next determined whether $\mathrm{Mcl}-1$ and survivin reduction is indeed involved in mediating induction of apoptosis by API-1. To this end, we enforced the expression of ectopic Mcl-1 or survivin in NSCLC cells and then examined their protective effects on API-1-induced apoptosis. In both $\mathrm{H} 1299$ and A549 cells, API-1 treatment caused clear cleavage of caspase-8, caspase-9, caspase- 3 and PARP in vector control cell lines; these effects were not observed or were drastically diminished in Mcl-1-transfected cell lines (Figure 2A). Moreover, API-1 did not or only minimally increased annexin V- positive (i.e., apoptotic) cell populations in both H1299/ Mcl-1 and A549/Mcl-1 cell lines, but drastically in their matched control counterparts (Figure 2B). These results clearly indicate that enforced expression of ectopic Mcl-1 protects cells from undergoing API-1-induced apoptosis, suggesting that $\mathrm{Mcl}-1$ reduction is indeed critical for mediating apoptosis induced by API-1. In contrast, enforced expression of ectopic survivin failed to protect cells from killing by API-1: survivin-transfected cells were even more sensitive than the control Lac Z-expressing cells to the effects of API-1 on PARP cleavage and cell survival (Figure 2C-D), suggesting that survivin reduction is less important than Mcl-1 in mediating induction of apoptosis by API-1.

We also asked whether $\mathrm{Mcl}-1$ reduction contributes to enhancement of TRAIL-induced apoptosis by API-1. We found that the combination of API-1 and TRAIL was far more active than either agent alone in decreasing
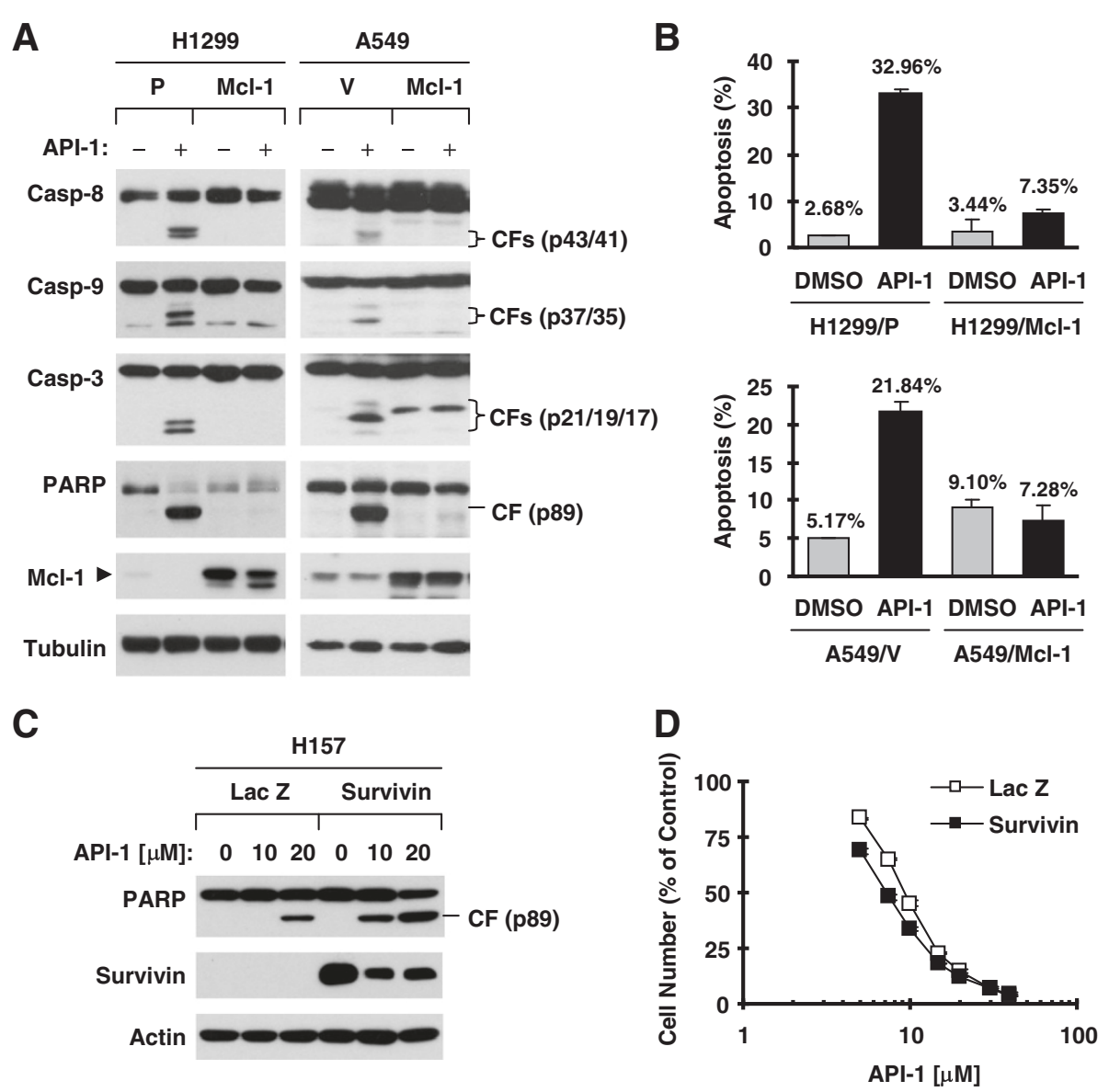

Figure 2 Enforced expression of ectopic Mcl-1 (A and B), but not survivin (C and D), protects cells from API-1-induced apoptosis including caspase activation. A and $\mathbf{B}$, The indicated stable transfectants were exposed to $5 \mu \mathrm{M}$ API-1 for $24 \mathrm{~h}$ and then harvested for preparation of whole-cell protein lysates and subsequent Western blot analysis to detect caspase cleavage and Mcl-1 expression (A) and for detection of apoptosis (i.e., annexin V-positive cells) with flow cytometry (B). P, parental; V, vector. C, The indicated cell lines were exposed to the given concentrations of API-1 for $24 \mathrm{~h}$ and then harvested for preparation of whole cell protein lysates and subsequent Western blot analysis. D, The indicated H157 stable transfectants were treated with different concentrations of API-1 as indicated for 3 days. Cell numbers were estimated with SRB assay. The data are means \pm SDs of four replicate determinations. CF, cleaved fragment. 
survival and inducing PARP cleavage in A549/V cells as we previously reported [2]; these effects were substantially diminished in A549/Mcl-1 cells (Figure 3). Hence, it is clear that enforced Mcl-1 expression protects cells from undergoing apoptosis by the API-1 and TRAIL combination, suggesting that $\mathrm{Mcl}-1$ reduction also contributes to augmentation of TRAIL-induced apoptosis by API-1. Collectively, these results provide a strong justification to focus our further studies on addressing the mechanisms by which API-1 induces Mcl-1 reduction.

\section{API-1 facilitates proteasomal degradation of $\mathrm{Mcl}-1$}

To elucidate the mechanisms by which API-1 reduces Mcl-1 levels, we detected Mcl-1 mRNA expression in cells exposed to different concentrations of API-1 with RT-PCR and found that API-1 did not alter Mcl-1 mRNA levels (Figure 4A). Since Mcl-1 is known to be a protein subject to proteasomal degradation [23,24], we next determined whether API-1-induced Mcl-1 reduction is due to enhancement of its degradation. Hence, we compared the effects of API-1 on Mcl-1 reduction in the absence and presence of the proteasome inhibitor MG132 in a few of NSCLC cell lines. API-1 effectively decreased Mcl-1 levels in the absence of MG132, but failed to do so in the presence of MG132 (Figure 4B), indicating that API-1 indeed induces proteasomal degradation of Mcl-1. Moreover, we found that the half-life of Mcl-1 in API-1-treated cells (about $20 \mathrm{~min}$ ) was shorter than that in DMSO-treated cells (about $90 \mathrm{~min}$ ) (Figure 4C), indicating that API-1 decreases the stability of Mcl-1. Taken together, we conclude that API-1 facilitates proteasomal degradation of $\mathrm{Mcl}-1$, resulting in Mcl-1 reduction.

\section{API-1 induces GSK3-dependent Mcl-1 degradation}

It is known that GSK3-mediated phosphorylation of Mcl-1 at S159 can trigger proteasomal degradation of Mcl-1 [8-11]. We therefore detected Mcl-1 phosphorylation in cells exposed to API-1. In API-1 sensitive H1299 and H522 cells, we detected a rapid and robust increase of p-Mcl-1 (S159/T163) early at $30 \mathrm{~min}$ post API-1 treatment followed by reduction of total levels of Mcl-1. In contrast, we detected a weak and late increase of p-Mcl-1 in API-1 insensitive H226 and H1792 cells (Figure 5A). Therefore, it is clear that API-1 increases Mcl-1 phosphorylation at S159, particularly in API-1 sensitive cell lines. We next determined whether API-1 induces a GSK3-dependent $\mathrm{Mcl}-1$ reduction. The presence of the chemical GSK3 inhibitor SB216763 inhibited Mcl-1 phosphorylation and reduction induced by API-1 in both H1299 and H522 cell lines (Figure 5B). In agreement, genetic suppression of GSK3 with GSK3 siRNA clearly rescued Mcl-1 reduction induced by API-1 (Figure 5C). Moreover, inhibition of GSK3 with SB216763 reversed the effect of API-1 on shortening the half-life of Mcl-1 or on decreasing the stability of Mcl-1 (Figure 5D). Together, it is apparent that API-1 induces a GSK3dependent $\mathrm{Mcl}-1$ reduction or degradation. To further demonstrate the requirement of GSK3-mediated S159 phosphorylation in API-1-induced Mcl-1 degradation, we compared the effects of API-1 on reducing the levels of ectopically expressed wild-type and mutant Mcl-1, in which serine 159 (S159) was changed into alanine (S159A). As presented in Figure 5E, API-1 effectively decreased the levels of wild-type Mcl-1, but did not alter the levels of Mcl-1 (S159A). Hence, it is clear that the phosphorylation of S159 in Mcl-1 is an essential step for API1-induced Mcl-1 degradation.

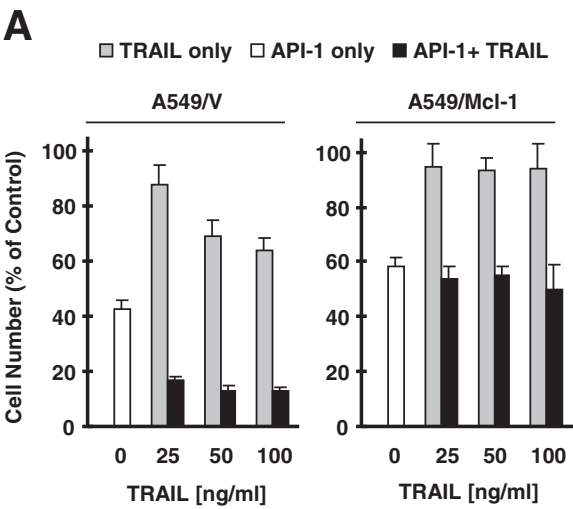

B

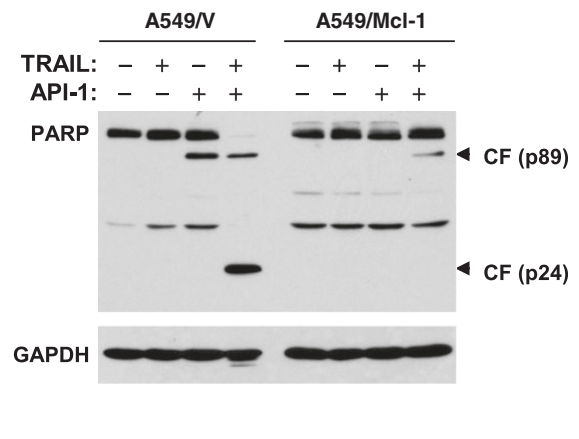

Figure 3 Figure Enforced expression of ectopic Mcl-1 in A549 cells protects cells from apoptosis induced by the API-1 and TRAIL combination. A, The indicated stable transfectants seeded in 96 well-plates were treated with $5 \mu \mathrm{M} \mathrm{API-1}$ alone, the indicated concentrations of TRAIL alone and API-1 in combination with TRAIL. After $24 \mathrm{~h}$, the cell numbers were estimated with SRB assay. The data are means \pm SDs of four replicate determinations. B, The indicated stable transfectants were treated with $5 \mu \mathrm{M} \mathrm{API-1}$ alone, $30 \mathrm{ng} / \mathrm{ml}$ TRAlL alone or their combination for $22 \mathrm{~h}$ and then harvested for preparation of whole-cell protein lysates and subsequent Western blot analysis to detect PARP cleavage. CF, cleaved form. 
A

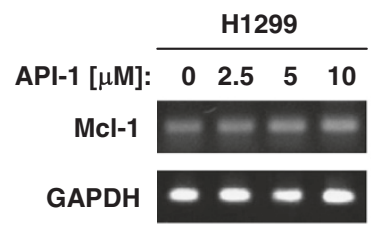

B

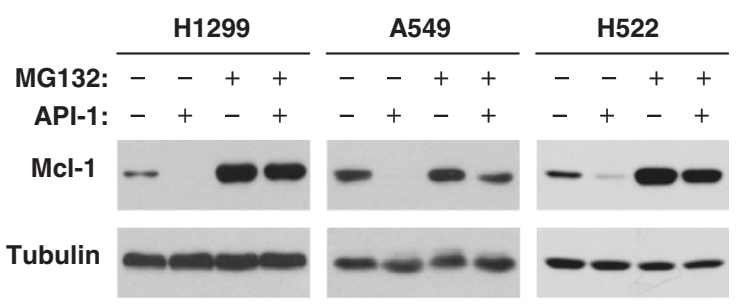

C
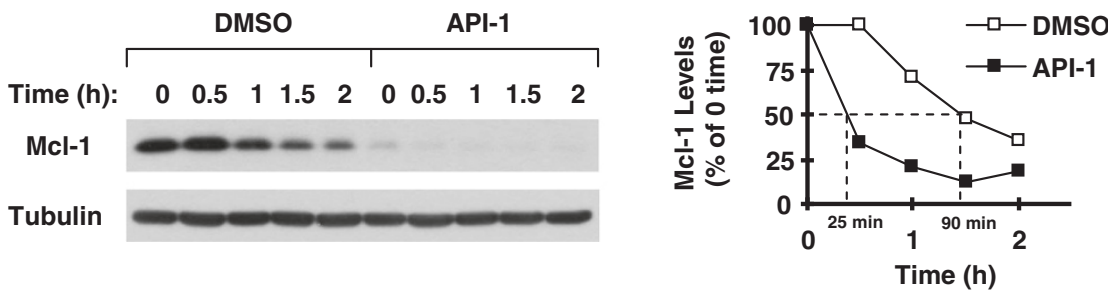

Figure 4 API-1 induces proteasome-mediated Mcl-1 degradation (B) and decreases Mcl-1 stability (C) without altering Mcl-1 mRNA levels (A). A, H1299 cells were exposed to the indicated concentrations of API- 1 for $8 \mathrm{~h}$. Total cellular RNA was then isolated from the cells for detection of Mcl-1 and GAPDH (internal control) mRNAs with RT-PCR. B, The indicated cell lines were pre-treated with $20 \mu \mathrm{M}$ MG132 for 30 minutes prior to the addition of $5 \mu \mathrm{M} \mathrm{API-1.} \mathrm{After} \mathrm{co-treatment} \mathrm{for} \mathrm{an} \mathrm{additional} 4 \mathrm{~h}$, the cells were harvested for preparation of whole-cell protein lysates and subsequent Western blot analysis. C, H1299 cells were treated with $5 \mu \mathrm{M} \mathrm{API-1}$ for 8 h. The cells were then washed with PBS 3 times and refed with fresh medium containing $10 \mu \mathrm{g} / \mathrm{ml} \mathrm{CHX.} \mathrm{At} \mathrm{the} \mathrm{indicated} \mathrm{times,} \mathrm{the} \mathrm{cells} \mathrm{were} \mathrm{harvested} \mathrm{for} \mathrm{preparation} \mathrm{of} \mathrm{whole-cell}$ protein lysates and subsequent Western blot analysis. Protein levels were quantified with NIH Image J software (Bethesda, MA) and were normalized to actin. The results were plotted as the relative Mcl-1 levels compared to those at the time 0 of $\mathrm{CHX}$ treatment (right panel).

\section{API-1 induces GSK3-dependent apoptosis}

If $\mathrm{Mcl}-1$ reduction is a key event that mediates induction of apoptosis, we anticipated that blockage of GSK3dependent Mcl-1 reduction will impair its ability to cause apoptosis. Hence, we further determined the impact of GSK3 inhibition on the ability of API-1 to induce apoptosis. By comparing the effects of API-1 on decreasing cell survival and inducing cleavage of caspases in the absence and presence of SB216763, we observed that cotreatment of cells with the API-1 and SB216763 combination was much weaker than API-1 alone in decreasing cell survival (Figure 6A) and in inducing cleavage of caspases including caspase-8, caspase-9, caspase- 3 and PARP (Figure 6B) in both $\mathrm{H} 1299$ and H522 cells. The CIs for most combinations were $>1$, suggesting antagonistic effects (Figure 6A). Thus, these data support the conclusion that API-1 also induces GSK3-dependent apoptosis.

The SKP1-Cul1-F-box protein (SCF) E3 ligases, $\beta$-TrCP and FBXW7, are involved in mediating $\mathrm{Mcl}-1$ degradation induced by API-1

Finally, we investigated which E3 ubiquitin ligase is responsible for the GSK3-dependent proteasomal degradation of Mcl-1 induced by API-1. Given the recent studies on the critical role of FBXW7 in mediating GSK3dependent degradation of Mcl-1 [10,11], we first determined whether this E3 ligase is involved in API-1-induced Mcl-1 degradation. Using the validated FBXW7 siRNA in our previous study [13], we detected slightly less reduction of Mcl-1 in FBXW7 siRNA-transfected cells than in control siRNA-transfected cells (Figure 7A). We further compared the effects of API-1 on Mcl-1 reduction between WT and FBXW7-KO HCT116 cells and generated similar results (Figure 7A) as we observed in the above knockdown experiment. These data collectively suggest that FBXW7 is only partly involved in mediating GSK3dependent degradation of Mcl-1 by API-1, and that additional E3 ligases(s) are also involved in this process.

$\beta-\operatorname{TrCP}$ was previously suggested to mediate GSK3dependent degradation of Mcl-1 [8]. We next determined whether this E3 ligase contributes to GSK3-dependent Mcl-1 degradation induced by API-1. Because both FBXW7 and $\beta$-TrCP are SCF E3 ligases and neddylation of Cul1 is required for the activity of SCF E3 ligases [25], we determined whether API-1 induces Mcl-1 degradation through a SCF E3 ligase-dependent mechanism. Inhibition of neddylation with MLN4924 impaired the ability of API-1 to decrease Mcl-1 levels (Figure 7B). In agreement, disruption of the SCF complex by knocking down of SKP1, Cul1 or both substantially rescued Mcl-1 reduction caused by API-1 (Figure 7C). These data clearly indicate the involvement of SCF E3 ligases in mediating API-1-induced Mcl-1 degradation. Following these experiments, we determined whether $\beta$-TrCP is involved in this process. As shown in Figure $7 \mathrm{C}$, knockdown of $\beta-\operatorname{TrCP}$ substantially attenuated the ability of API- 1 to decrease Mcl-1 levels, as did knockdown of Cul1 


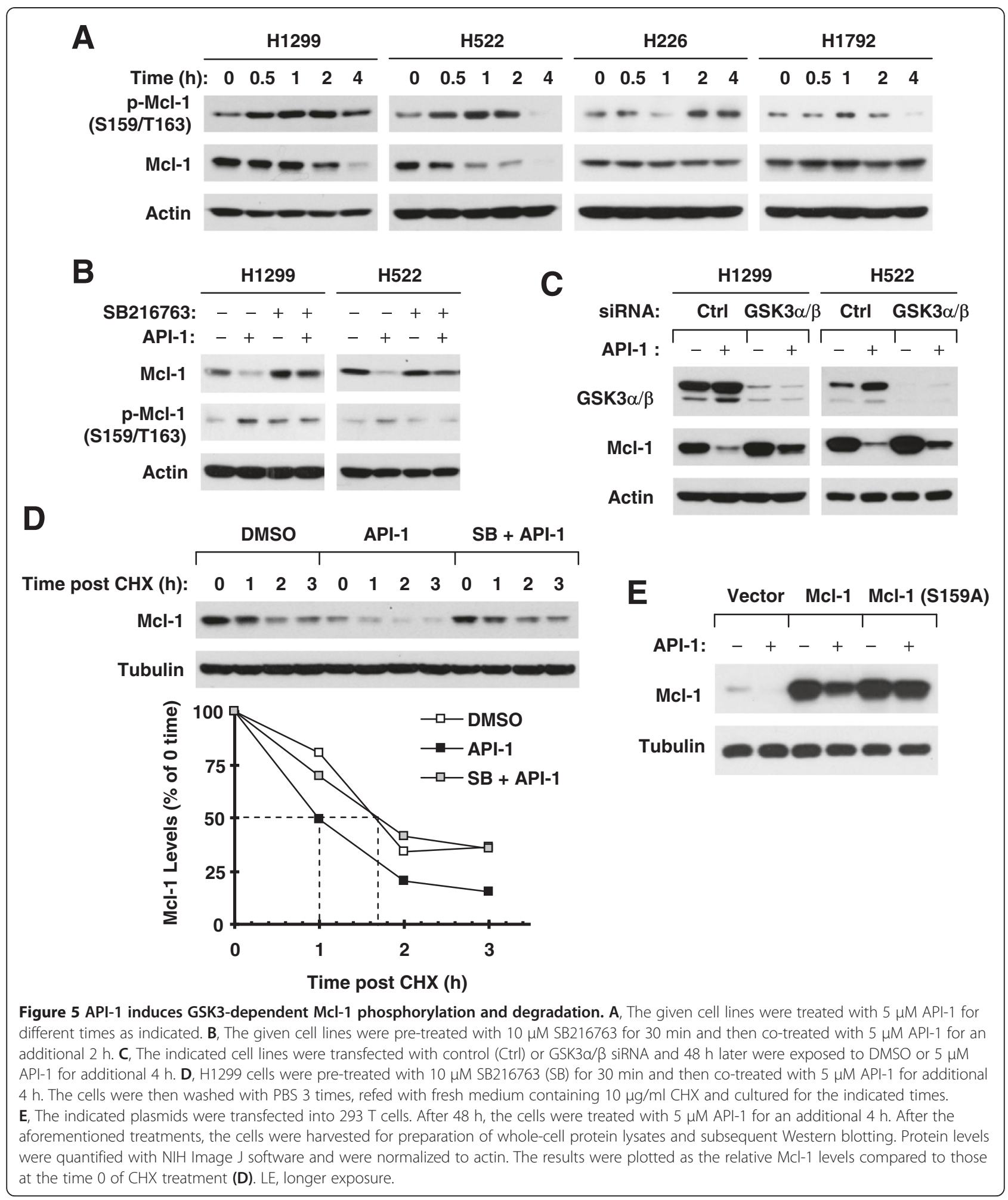

or SKP1, indicating that $\beta$-TrCP is indeed involved in mediating Mcl-1 degradation induced by API-1. Compared to the effects of $\beta$-TrCP or FBXW7 knockdown alone, coknockdown of both $\beta$-TrCP and FBXW7 enhanced the rescuing effect on $\mathrm{Mcl}-1$ reduction induced by API-1
(Figure 7D). The knockdown efficiencies of $\beta$-TrCP siRNA, FBXW7 siRNA or their combination were evaluated with RT-PCR (Figure 7E). Collectively, we suggest that both $\beta$-TrCP and FBXW7 are responsible for GSK3dependent degradation of Mcl-1 induced by API-1. 

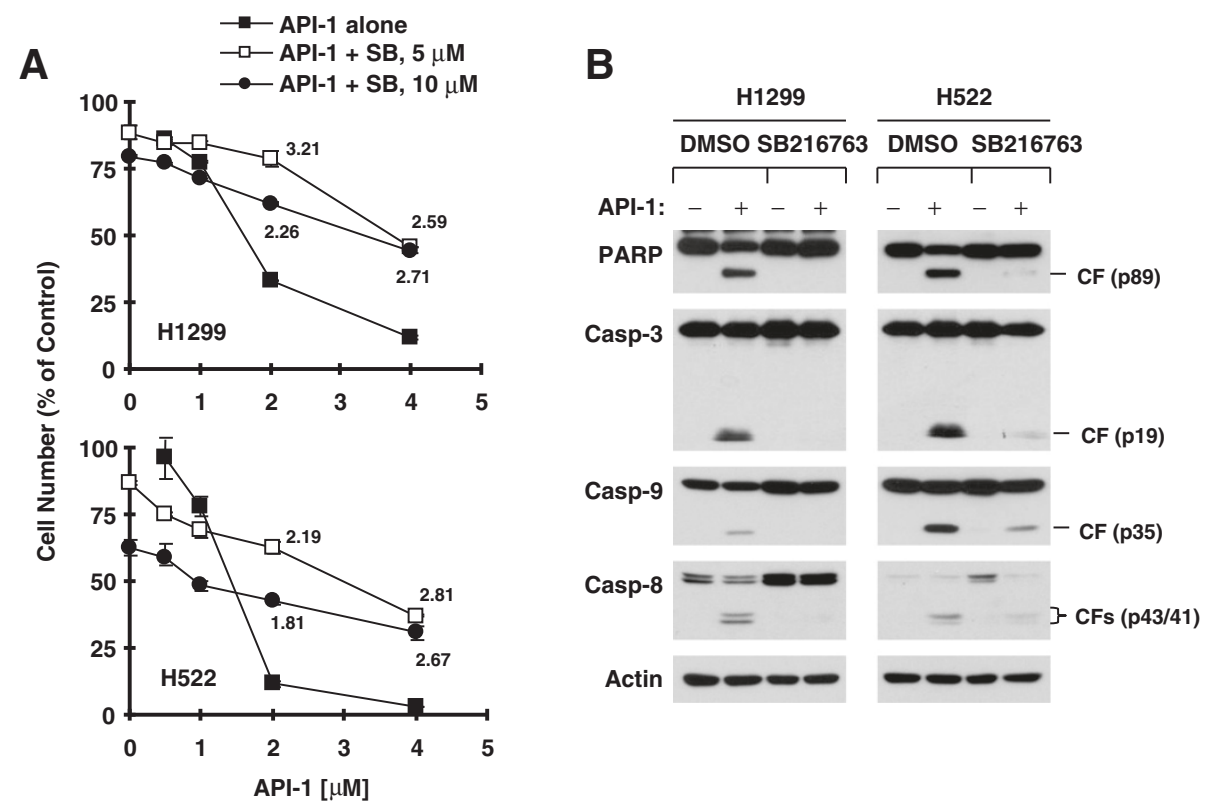

Figure 6 Inhibition of GSK3 with SB216763 antagonizes the effects of API-1 on decreasing cell survival (A) and inducing caspase cleavage (B). A, The indicated cell lines were exposed to different concentrations of API-1 alone, SB216763 alone and the combination of API-1 and SB216763. After 3 days, cell numbers were estimated with the SRB assay. Data are means \pm SDs of four replicate determinations. The numbers

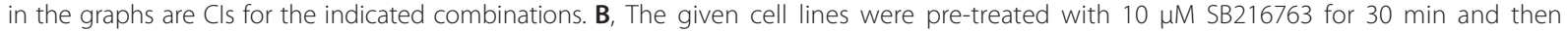
co-treated with $5 \mu \mathrm{M} \mathrm{API}-1$ for $24 \mathrm{~h}$ and then harvested for preparation of whole-cell protein lysates and subsequent Western blotting. $C F$, cleaved form.

\section{Discussion}

Mcl-1 is a well-known Bcl-2 family protein that negatively regulates apoptosis by binding and sequestering the pro-apoptotic proteins such as Bax, Bak, Noxa, and Bim [7]. In this study, we found that API-1 rapidly and potently decreased Mcl-1 levels in NSCLC cell lines sensitive to API-1, but only minimally in API-1-insenstive cell lines. Moreover, enforced expression of ectopic Mcl-1 substantially protected NSCLC cells from undergoing apoptosis induced by API-1. These results clearly demonstrated that $\mathrm{Mcl}-1$ reduction is an essential event for API1 to induce apoptosis. In this study, API-1 also reduced the levels of survivin, another important anti-apoptotic protein that acts downstream of Mcl-1 as an endogenous inhibitor of caspases (e.g., caspase-9) [26]. However, we failed to demonstrate its essential role in mediating induction of apoptosis by API-1 because API-1 decreased survivin levels in NSCLC cells regardless of their sensitivities to API-1 and was equally effective in killing both NSCLC cells carrying a vector control and those expressing ectopic survivin. Hence the finding of Mc1 reduction as a critical mechanism accounting for API-1-induced apoptosis is novel. The current study focuses on demonstrating the role of Mcl-1 suppression in API-1-induced apoptosis. However, this does not exclude other targets or mechanisms such as Bcl-1 reduction described in this study (Figure 1) and c-FLIP degradation reported previously [2] that may account for API-1-induced apoptosis, particularly in a given cancer cell line. It is more likely that the effect of API-1 on induction of apoptosis is an outcome of the combination of multiple mechanisms including $\mathrm{Mcl}-1$ reduction.

We noted that enforced expression of ectopic Mcl-1 blocked cleavage of not only caspase-9, but also caspase8 . It is very likely that caspase- 8 activation caused by API-1 is secondary to activation of the intrinsic apoptotic pathway because caspase- 8 can be activated by caspase- 9 or caspase-3 $[27,28]$. However, we currently cannot rule out the possibility that caspase- 8 activation is caused by Mcl-1 suppression if Mcl-1 has an uncovered role in direct suppression of the extrinsic apoptotic pathway.

We previously reported that API-1 effectively enhances TRAIL-induced apoptosis in human NSCLC cells involving induction of c-FLIP degradation [2]. In this study, we found that enforced expression of ectopic Mcl-1 protected NSCLC cells from induction of apoptosis by the combination of API-1 and TRAIL. Hence, it is apparent that Mcl1 reduction is also an important mechanism by which API-1 augments TRAIL-induced apoptosis.

Mcl-1 is known to undergo GSK3-dependent proteasomal degradation [7,23]. As an Akt inhibitor, it was plausible to speculate that API-1 reduces Mcl-1 levels through activating GSK3 followed by enhancing GSK3- 


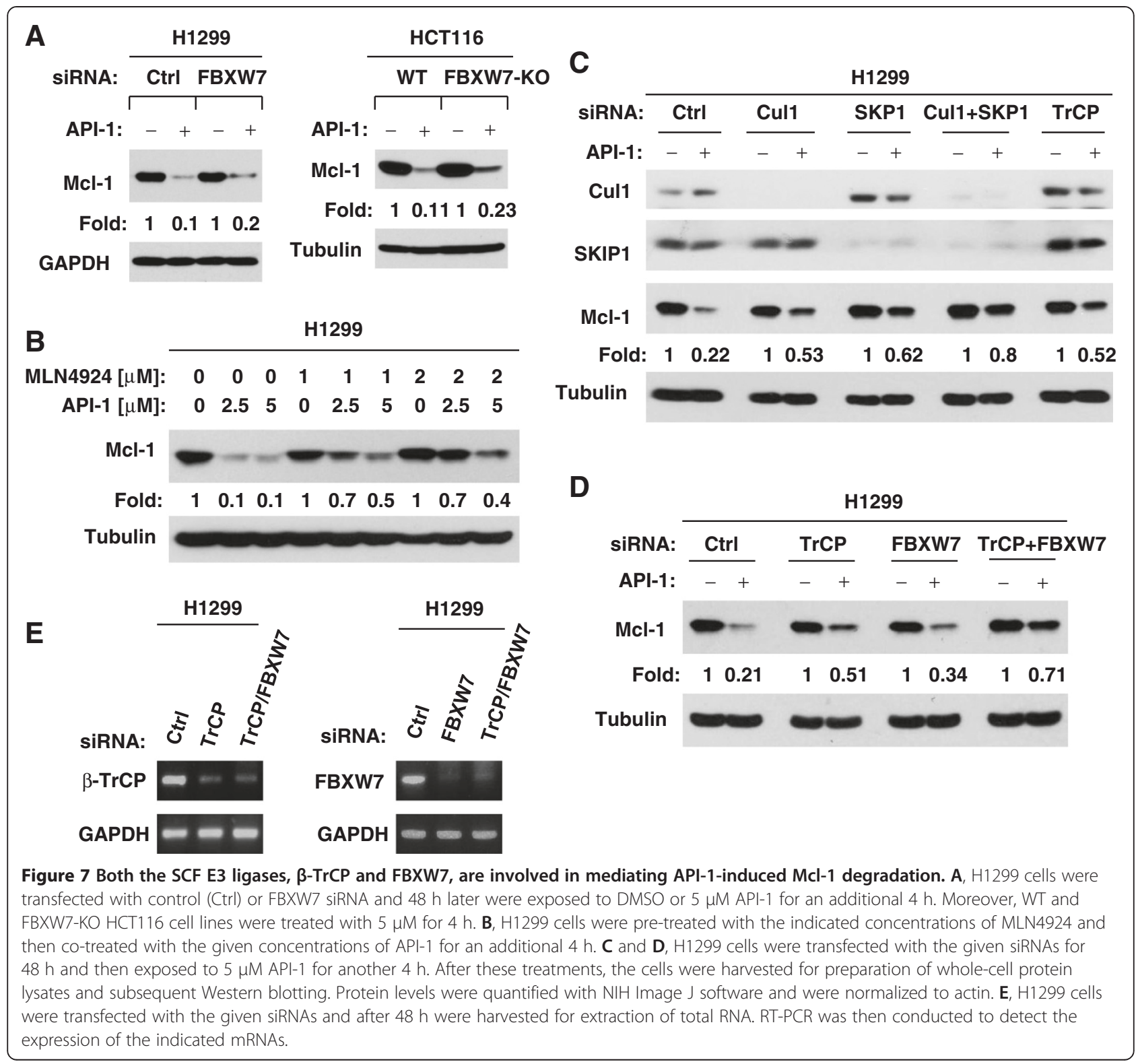

dependent Mcl-1 degradation. In this study, API-1 did not alter Mcl-1 mRNA levels, suggesting that API-1induced $\mathrm{Mcl}-1$ reduction is likely to be a posttranscriptional event. Indeed, inhibition of proteasome with MG132 rescued $\mathrm{Mcl}-1$ reduction induced by API-1. Moreover, API-1 decreased Mcl-1 stability. Hence, it is clear that API-1 induces proteasomal degradation of Mcl-1, leading to $\mathrm{Mcl}-1$ reduction. We observed that decreased Mcl-1 levels were accompanied with an early and robust increase in Mcl-1 phosphorylation at S159/T163, primarily in in those NSCLC cell lines sensitive to API-1. Importantly, suppression of GSK3 inhibited Mcl-1 phosphorylation and reduction or degradation induced by API-1. In agreement, API-1 lost its activity on inducing degradation of mutant Mcl-1 (S159A), in which S159 could not be phosphorylated. Collectively, we conclude that API-1 decreases Mcl-1 levels through facilitating GSK3-dependent proteasomal degradation of Mcl-1.

In our study, we found that inhibition of GSK3 (e.g., with SB216763) antagonized the effects of API-1 on decreasing the survival of NSCLC cells and on inducing cleavage of caspases and PARP. Thus, it is clear that API-1 induces GSK3-dependent apoptosis as well. This reinforces the critical role of GSK3-dependent Mcl-1 reduction in mediating apoptosis induced by API-1. This finding also implies that, to prevent potential antagonism, API-1 should not be used in combination with any agents that may inhibit GSK3 activity in the treatment of cancer.

Although FBXW7 has been recently suggested to be a key E3 ligase that mediates GSK3-dependent Mcl-1 
degradation [10,11], we found that knockdown or knockout of FBXW7 provided only limited protective effect against Mcl-1 reduction induced by API- 1 . However, this process does require activation of SCF E3 ligases since the inhibition of SCF complex formation by knockdown of Cul1, SKP1 or both drastically impaired the ability of API-1 to decrease Mcl-1 levels. Hence, FBXW7 may not be the major E3 ligase responsible for API-1-induced GSK3-dependent proteasomal degradation of Mcl-1, and additional SCF E3 ligase(s) may be involved in mediating GSK3-dependent degradation of Mcl-1 induced by API-1. Indeed, our further studies have shown that $\beta$-TrCP, another SCF E3 ligase involved in mediating GSK3-dependent degradation of Mcl-1 as suggested previously [8], also contributes to API1 -induced Mcl-1 degradation since knockdown of $\beta$-TrCP provided a more drastic effect than FBXW7 knockdown on rescuing Mcl-1 reduction induced by API-1. Moreover, we found that co-knockdown of both $\beta$-TrCP and FBXW7 exhibited much more potent effects than knockdown of either single gene in preventing Mcl-1 reduction induced by API-1. Therefore, we believe that both $\beta$-TrCP and FBXW7 are involved in mediating GSK3-dependent Mcl-1 degradation induced by API-1. Our findings clearly suggest that two E3 ubiquitin ligases can cooperate to regulate the degradation of one protein.

\section{Conclusions}

The current study has demonstrated that API-1 decreases Mcl-1 levels through facilitating GSK3-dependent, $\beta$-TrCP and FBXW7-mediated protein degradation. By reducing Mcl-1 levels, API-1 is able to induce apoptosis and sensitize cancer cells to TRAIL-induced apoptosis.

\section{Abbreviations}

API-1: 4-amino-5,8-dihydro-5-oxo-8- $\beta-D-r i b o f u r a n o s y l-p y r i d o[2,3-d]$ pyrimidine6-carboxamide; TRAIL: Tumor necrosis factor-related apoptosis-inducing ligand; NSCLC: Non-small cell lung cancer; GSK3: Glycogen synthase kinase 3; $\beta-\operatorname{TrCP}: \beta-$ Transducin repeats-containing protein; FBXW7: F-box/WD repeatcontaining protein 7; WT: Wild-type; GAPDH: Glyceraldehyde 3-phosphate dehydrogenase; CHX: Cycloheximide; SRB: Sulforhodamine B;

Cl: Combination index; siRNA: Small interfering RNA; Cul1: Cullin1;

RT-PCR: Reverse transcription-PCR; SCF: SKP1-cullin1-F-box protein.

\section{Competing interests}

All authors declare that they have no competing interests.

\section{Authors' contributions \\ $H R, J K, B G$ and PY designed and conducted experiments and data analysis. $X D, M C$ and FRK participated in discussion of the data. XD provided some critical reagents. MC and SYS participated in experimental design, coordination, data analysis and draft of the manuscript. All authors read and approved the final manuscript.}

\section{Authors' information}

$\mathrm{H}$ Ren, J Koo and B Guan share the first authorship.

\section{Acknowledgements}

We thank Dr. A. Hammond in our department for editing the manuscript and Dr. B. Vogelstein for providing FBXW7-deficient cells. We are also grateful to the high school students, Kevin Sun and Jerry Yue, from Parkview High
School (Lilburn, GA) for assisting performance of some experiments. This study was supported by NIH R01 CA118450 (S-Y Sun) and R01 CA160522 (S-Y Sun). FR Khuri and S-Y Sun are Georgia Cancer Coalition Distinguished Cancer Scholars.

\section{Author details}

${ }^{1}$ Department of Respiration, First Affiliated Hospital of Medical College of Xi'an Jiaotong University, Xi'an, Shaanxi, P R China. ${ }^{2}$ Department of Hematology and Medical Oncology, Emory University School of Medicine and Winship Cancer Institute, Atlanta, Georgia, USA. ${ }^{3}$ Department of Radiation Oncology, Emory University School of Medicine and Winship Cancer Institute, Atlanta, Georgia, USA.

Received: 29 August 2013 Accepted: 18 November 2013 Published: 22 November 2013

\section{References}

1. Kim D, Sun M, He L, Zhou QH, Chen J, Sun XM, Bepler G, Sebti SM, Cheng JQ: A small molecule inhibits Akt through direct binding to Akt and preventing Akt membrane translocation. J Biol Chem 2010, 285:8383-8394.

2. Li B, Ren H, Yue P, Chen M, Khuri FR, Sun SY: The novel Akt inhibitor API-1 induces c-FLIP degradation and synergizes with TRAIL to augment apoptosis independent of Akt inhibition. Cancer Prev Res (Phila) 2012, 5:612-620.

3. Hengartner MO: The biochemistry of apoptosis. Nature 2000, 407:770-776.

4. Shirley S, Micheau O: Targeting c-FLIP in cancer. Cancer Lett 2013, 332:141-150.

5. Chan SL, Yu VC: Proteins of the bcl-2 family in apoptosis signalling: from mechanistic insights to therapeutic opportunities. Clin Exp Pharmacol Physiol 2004, 31:119-128.

6. Zhang L, Fang B: Mechanisms of resistance to TRAlL-induced apoptosis in cancer. Cancer Gene Ther 2005, 12:228-237.

7. Thomas LW, Lam C, Edwards SW: MCl-1; the molecular regulation of protein function. FEBS Lett 2010, 584:2981-2989.

8. Ding Q, He X, Hsu JM, Xia W, Chen CT, Li LY, Lee DF, Liu JC, Zhong Q, Wang $X$, Hung MC: Degradation of Mcl-1 by beta-TrCP mediates glycogen synthase kinase 3-induced tumor suppression and chemosensitization. Mol Cell Biol 2007, 27:4006-4017.

9. Maurer U, Charvet C, Wagman AS, Dejardin E, Green DR: Glycogen synthase kinase- 3 regulates mitochondrial outer membrane permeabilization and apoptosis by destabilization of MCL-1. Mol Cell 2006, 21:749-760.

10. Wertz IE, Kusam S, Lam C, Okamoto T, Sandoval W, Anderson DJ, Helgason E, Ernst JA, Eby M, Liu J, Belmont LD, Kaminker JS, O'Rourke KM, Pujara K, Kohli PB, Johnson AR, Chiu ML, Lill JR, Jackson PK, Fairbrother WJ, Seshagiri S, Ludlam MJ, Leong KG, Dueber EC, Maecker H, Huang DC, Dixit VM: Sensitivity to antitubulin chemotherapeutics is regulated by MCL1 and FBW7. Nature 2011, 471:110-114.

11. Inuzuka H, Shaik S, Onoyama I, Gao D, Tseng A, Maser RS, Zhai B, Wan L, Gutierrez A, Lau AW, Xiao Y, Christie AL, Aster J, Settleman J, Gygi SP, Kung AL, Look T, Nakayama Kl, DePinho RA, Wei W: SCF(FBW7) regulates cellular apoptosis by targeting MCL1 for ubiquitylation and destruction. Nature 2011, 471:104-109.

12. Ren H, Chen M, Yue P, Tao H, Owonikoko TK, Ramalingam SS, Khuri FR, Sun SY: The combination of RAD001 and NVP-BKM120 synergistically inhibits the growth of lung cancer in vitro and in vivo. Cancer Lett 2012, 325:139-146.

13. Ren H, Zhao L, Li Y, Yue P, Deng X, Owonikoko TK, Chen M, Khuri FR, Sun SY: The PI3 kinase inhibitor NVP-BKM120 induces GSK3/FBXW7dependent $\mathrm{Mcl}-1$ degradation, contributing to induction of apoptosis and enhancement of TRAlL-induced apoptosis. Cancer Lett 2013, 338:229-238.

14. Lin Y, Liu X, Yue P, Benbrook DM, Berlin KD, Khuri FR, Sun SY: Involvement of c-FLIP and survivin down-regulation in flexible heteroarotinoidinduced apoptosis and enhancement of TRAIL-initiated apoptosis in lung cancer cells. Mol Cancer Ther 2008, 7:3556-3565.

15. Sun SY, Yue P, Dawson MI, Shroot B, Michel S, Lamph WW, Heyman RA, Teng M, Chandraratna RA, Shudo K, Hong WK, Lotan R: Differential effects of synthetic nuclear retinoid receptor-selective retinoids on the growth of human non-small cell lung carcinoma cells. Cancer Res 1997, 57:4931-4939. 
16. Liu X, Yue P, Zhou Z, Khuri FR, Sun SY: Death receptor regulation and celecoxib-induced apoptosis in human lung cancer cells. J Natl Cancer Inst 2004, 96:1769-1780.

17. Sun SY, Yue P, Wu GS, El-Deiry WS, Shroot B, Hong WK, Lotan R: Mechanisms of apoptosis induced by the synthetic retinoid CD437 in human non-small cell lung carcinoma cells. Oncogene 1999, 18:2357-2365.

18. Mao JH, Perez-Losada J, Wu D, Delrosario R, Tsunematsu R, Nakayama KI, Brown K, Bryson S, Balmain A: Fbxw7/Cdc4 is a p53-dependent, haploinsufficient tumour suppressor gene. Nature 2004, 432:775-779.

19. Yang W, Xia Y, Ji H, Zheng Y, Liang J, Huang W, Gao X, Aldape K, Lu Z: Nuclear PKM2 regulates beta-catenin transactivation upon EGFR activation. Nature 2011, 480:118-122.

20. Yokobori T, Mimori K, Iwatsuki M, Ishii H, Tanaka F, Sato T, Toh H, Sudo T, Iwaya T, Tanaka Y, Onoyama I, Kuwano H, Nakayama Kl, Mori M: Copy number loss of FBXW7 is related to gene expression and poor prognosis in esophageal squamous cell carcinoma. Int J Oncol 2012, 41:253-259.

21. Liu Z, Liu M, Niu G, Cheng Y, Fei J: Genome-wide identification of target genes repressed by the zinc finger transcription factor REST/NRSF in the HEK 293 cell line. Acta Biochim Biophys Sin (Shanghai) 2009, 41:1008-1017.

22. Li R, Hu Z, Sun SY, Chen ZG, Owonikoko TK, Sica GL, Ramalingam SS, Curran WJ, Khuri FR, Deng X: Niclosamide Overcomes Acquired Resistance to Erlotinib through Suppression of STAT3 in Non-Small Cell Lung Cancer. Mol Cancer Ther 2013, 12:2200-2212.

23. Inuzuka H, Fukushima H, Shaik S, Liu P, Lau AW, Wei W: Mcl-1 ubiquitination and destruction. Oncotarget 2011, 2:239-244.

24. Warr MR, Shore GC: Unique biology of Mcl-1: therapeutic opportunities in cancer. Curr Mol Med 2008, 8:138-147.

25. Jia L, Sun Y: SCF E3 ubiquitin ligases as anticancer targets. Curr Cancer Drug Targets 2011, 11:347-356.

26. Altieri DC: Validating survivin as a cancer therapeutic target. Nat Rev Cancer 2003, 3:46-54.

27. Viswanath V, Wu Y, Boonplueang R, Chen S, Stevenson FF, Yantiri F, Yang L, Beal MF, Andersen JK: Caspase-9 activation results in downstream caspase-8 activation and bid cleavage in 1-methyl-4-phenyl-1,2,3,6tetrahydropyridine-induced Parkinson's disease. J Neurosci 2001, 21:9519-9528.

28. Ferreira KS, Kreutz C, Macnelly S, Neubert K, Haber A, Bogyo M, Timmer J, Borner C: Caspase-3 feeds back on caspase-8, Bid and XIAP in type I Fas signaling in primary mouse hepatocytes. Apoptosis 2012, 17:503-515.

doi:10.1186/1476-4598-12-146

Cite this article as: Ren et al: The E3 ubiquitin ligases $\beta$-TrCP and FBXW7 cooperatively mediates GSK3-dependent Mcl-1 degradation induced by the Akt inhibitor API-1, resulting in apoptosis. Molecular Cancer 2013 12:146.

\section{Submit your next manuscript to BioMed Central and take full advantage of:}

- Convenient online submission

- Thorough peer review

- No space constraints or color figure charges

- Immediate publication on acceptance

- Inclusion in PubMed, CAS, Scopus and Google Scholar

- Research which is freely available for redistribution 